\title{
Selenium depletion modulates the expression of the protein biosynthetic pathway in Caco-2 cells
}

\author{
A. Maciel Domínguez, D. Swan, D. Ford and J. Hesketh \\ Institute for Cell and Molecular Biosciences and Human Nutrition Research Centre, Faculty of Medical Sciences, Newcastle \\ University, Framlington Place, Newcastle-upon-Tyne, NE2 4HH, UK
}

Selenium (Se) is a micronutrient with an important role in health and disease processes. Increased Se intake $(100-200 \mu \mathrm{g} / \mathrm{d}) \mathrm{has}$ shown to enhance immunity ${ }^{(1)}$, promote antioxidant defence and lower risk of mortality from colorectal cancer ${ }^{(2)}$. Recent transcriptomic studies have shown that changes in Se intake not only alter the expression of the Se-containing selenoproteins but also affect the expression of downstream targets ${ }^{(3,4)}$. The protein biosynthetic pathways showed altered expression in both human lymphocytes ${ }^{(3)}$ and the mouse colon $^{(4)}$. The aim of the present work was to investigate whether in a gut epithelial cell line the genes expressing components of the protein biosynthetic pathway are altered in expression during Se depletion and whether miRNA are involved in the regulation of this pathway by Se. Three algorithms (mirBASE, microrna.org and GeneSet2miRNA) and ingenuity pathway analysis were used to identify potential miRNA targets in the protein biosynthetic pathway. From these targets, four genes (RPL24, MRPS36, EIF4B, EIF4EBP2) were identified by at least two bioinformatic tools and their expression in response to Se depletion was assessed in the gut epithelial cell line Caco-2 by semi-quantitative RT-PCR. Caco-2 cells were grown for $72 \mathrm{~h}$ in Dulbecco Modified Eagle's medium with or without sodium selenite $(7 \mathrm{ng} / \mathrm{ml} ; 40.5 \mathrm{~nm})$ and the total RNA was extracted. Semi-quantitative RT-PCR analysis showed that the expression of selenoprotein W and glutathione peroxidase 1 was lowered in cells grown in the Se-deficient medium, confirming the low cell Se-status ${ }^{(5)}$; the mean (SEM) selenoprotein W expression was $100 \%$ (3.7) and $41 \%$ (1.8) in cells grown in Se-supplemented and Se-deficient medium, respectively ( $n 3, P<0.005)$, and glutathione peroxidase 1 expression was $100 \%$ (3.0) and $42 \%(1.4)$, respectively $(n 3, P<0.005)$. The mean (SEM) expression of the potential target genes was increased in cells grown in an Se-deficient medium compared with cells grown in a supplemented medium: EIF4EBP2, $158 \%$ (3.4) and 100\% (3.9); EIF4B, 149\% (3.0) and 100\% (2.6); RPL24, 148\% (2.2) and 100\% (1.3); MRPS36, $186 \%$ (3.9) and $100 \%$ (2.2) (n 3, P<0.002). These results indicate that Se supply alters the expression of genes within the protein biosynthetic pathway, possibly through miRNA regulation. In future experiments, quantitative real-time PCR will be used to confirm these effects and miRNA expression after Se depletion will be assessed using a microarray approach.

A.M.D. was supported by The Consejo Nacional de Ciencia y Tecnología (CONACYT).

1. Arthur JR, McKenzie RC \& Beckett GJ (2003) Selenium in the immune system. J Nutr 133, Suppl. 1, 1457S-1459S.

2. Whanger PD (2004) Selenium and its relationship to cancer: an update. Br J Nutr 91, 11-28.

3. Pagmantidis VMC, van Schothorst EM, Keijer J et al. (2008) Supplementation of healthy volunteers with nutritionally relevant amounts of selenium increases the expression of lymphocyte protein biosynthesis genes. Am J Clin Nutr 87, 181-189.

4. Kipp A, Banning A, van Schothorst EM et al. (2009) Four selenoproteins, protein biosynthesis, and Wnt signalling are particularly sensitive to limited selenium intake in mouse colon. Mol Nutr Food Res 53, 1561-1572.

5. Pagmantidis V, Bermano G, Villette S et al. (2005) Effects of Se-depletion on glutathione peroxidase and selenoprotein W gene expression in the colon. FEBS Lett 579, 792-796. 